\title{
Perfil e demandas de saúde de adolescentes escolares ${ }^{I}$
}

Profile and health demands of school-age adolescents

Perfil y demanda de salud de adolescentes escolares

\section{Gabriela Aparecida da Silva ${ }^{\text {II }}$ Isabely Karoline da Silva Ribeiro ${ }^{I I}$, Hugo Ricardo Moreira da Silva ${ }^{\mathrm{I}}$, Tércia Maria Ribeiro Lima Rezendev ${ }^{\mathrm{V}}$ Vinícius Silva Belovi \\ Márcia Christina Caetano Romano ${ }^{\mathrm{VII}}$}

Resumo: Objetivo: identificar o perfil e as demandas de saúde de adolescentes escolares no Município de Divinópolis, Minas Gerais. Método: estudo transversal, exploratório-descritivo, realizado com 389 adolescentes do $8^{\circ}$ e 9o anos do ensino fundamental. Resultados: entre os participantes do estudo, $34,4 \%$ já fizeram uso de álcool, $17,7 \%$ experimentaram fumar e 7,4\% fizeram uso de maconha. A maioria dos adolescentes considerou seu estado de saúde como ótimo ou bom (84,4\%). Dos que iniciaram atividade sexual, $28,9 \%$ relataram não utilizar nenhum tipo de método. Percentual elevado de adolescentes mencionou solidão em algum momento da vida $(77,9 \%)$ e $5,3 \%$ dos meninos envolveram-se em brigas. Conclusão: as demandas dos adolescentes relacionadas ao sexo inseguro, saúde mental fragilizada, uso de álcool e outras drogas foram identificadas como prioritárias, necessitando da atuação conjunta entre saúde e educação na construção de estratégias comuns para o atendimento das necessidades inerentes ao adolescente.

Descritores: Vulnerabilidade em Saúde; Adolescente; Necessidades e Demandas de Serviços de saúde; Sexualidade; Estudos Transversais

\footnotetext{
${ }^{\text {I }}$ Artigo extraído do Trabalho de Conclusão de Curso intitulado "Necessidades de saúde de adolescentes do município de Divinópolis - Minas Gerais”, Curso de Enfermagem da Universidade Federal de São João Del-Rei, Campus Centro-Oeste, 2018

II Enfermeira pela Universidade Federal de São João Del-Rei - UFSJ. Divinópolis, Minas Gerais, Brasil. gabrielasilva-ufsj@hotmail.com. ORCID: https://orcid.org/0000-0003-1070-7141

III Enfermeira. Mestranda em Enfermagem pelo Programa de Pós-Graduação Mestrado Acadêmico em Enfermagem da Universidade Federal de São João Del-Rei - UFSJ. Divinópolis, Minas Gerais, Brasil. isabelykaroline@hotmail.com. ORCID: https://orcid.org/0000-0003-0732-7631

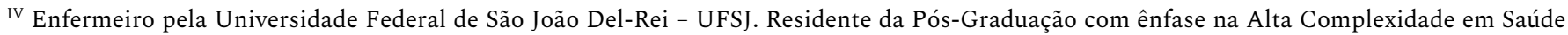
no Hospital Universitário da Universidade Federal de Santa Catarina - UFSC. Florianópolis, Santa Catarina, Brasil. hugorisilva@gmail.com. ORCID: https://orcid.org/0000-0001-7506-0508

$\checkmark$ Enfermeira. Mestrado em Enfermagem pela Escola de Enfermagem da Universidade Federal de Minas Gerais - UFMG. Secretaria Municipal de Saúde de Belo Horizonte/Diretoria Regional de Saúde Pampulha. Belo Horizonte, Minas Gerais, Brasil. terciamarili@hotmail.com. https://orcid.org/0000-0001-7731-011X

VI Doutor. Professor Adjunto da Universidade Federal de São João Del-Rei - UFSJ. Divinópolis, Minas Gerais, Brasil. viniciusbelo4@hotmail.com. http://orcid.org/0000-0003-0183-1175

VII Enfermeira. Doutora. Professora Associada da Universidade Federal de São João Del-Rei - UFSJ. Divinópolis, Minas Gerais, Brasil. marciachristinacs@gmail.com. http://orcid.org/0000-0002-1819-4689.
} 


\begin{abstract}
Objective: to identify the profile and health demands of school-age adolescents in Divinopolis, Minas Gerais, Brazil. Method: cross-sectional, exploratory-descriptive study, developed with 389 adolescents from the 8th and 9th grades of elementary school. Results: Among the study participants, $34.4 \%$ had already consumed alcohol, $17.7 \%$ had tried smoking and $7.4 \%$ marijuana. Most of the adolescents considered their health status as excellent or good (84.4\%). Among the students who were sexually active, $28.9 \%$ reported not using any type of contraceptive method. Many adolescents (77.9\%) mentioned loneliness at some point in their lives and 5.3\% of the boys had engaged in fights. Conclusion: the adolescents' priority demands were related to unsafe sex, impaired mental health, alcohol and drug use, demanding the joint action of health and education in the construction of shared strategies for meeting the adolescents' inherent needs.
\end{abstract}

Descriptors: Vulnerability in Health; Teenager; Needs and demands of health services; Sexuality; Cross-Sectional Studies

Resumen: Objetivo: identificar el perfil y las demandas de salud de adolescentes escolares en el município de Divinopolis, Minas Gerais, Brasil. Método: Estudio transversal, desarrollado con 389 adolescentes del 8o y 9o año de la enseñanza fundamental. Resultados: Entre los participantes, 34,4\% consumió alcohol al menos una vez, 17,7\% experimentó fumar y 7,4\% usó marihuana. La mayoría de los adolescentes consideró su estado de salud como óptimo o bueno (84,4\%). De los que iniciaron actividad sexual, el $28,9 \%$ relató no utilizar ningún tipo de método anticonceptivo. Muchos adolescentes $(77,9 \%)$ mencionaron soledad en algún momento de la vida y el 5,3\% de los niños se involucró en peleas. Conclusión: Los asuntos relacionados con la sexualidad, salud mental, uso de alcohol y otras drogas se identificaron como prioridades, que requieren la acción conjunta entre la salud y la educación en la construcción de estratégias comunes para satisfacer las necessidades inherentes de los adolescente.

Descriptores: Vulnerabilidad en Salud; Adolescentes; Necesidades y Demandas de Servicios de Salud; Sexualidad; Estudios Transversales

\title{
Introdução
}

As demandas de saúde passaram a ser objeto de estudo, debates e políticas públicas, podendo ser constituídas por indicadores que sinalizam possíveis vulnerabilidades em que as populações se encontram. Assim, as demandas de saúde auxiliam na sensibilização dos gestores de saúde e no direcionamento das políticas e programas públicos de saúde. ${ }^{1}$ A vulnerabilidade pode ser definida como a formação de vínculo frágil ou mesmo inexistente, que relaciona a afetividade do indivíduo à esfera social. Constitui-se em fragilidades e desigualdades de acesso a bens e serviços públicos. ${ }^{2-3}$ Desta forma, a vulnerabilidade permeia todo o ciclo vital do ser humano.

Dentre as vulnerabilidades presentes na adolescência pode-se eleger, por exemplo, aquelas que se destacaram em um estudo que descreveu o perfil dos jovens no âmbito nacional 
brasileiro, tais como o consumo indevido de álcool (23,8\%); tabaco (5,6\%); de drogas ilícitas (9\%); a alimentação não saudável (26,7\%); a redução do tempo de atividade física $(60,8 \%)$; o tempo de tela superior a duas horas (60\%); e a difícil relação intrafamiliar com a presença de problemas de saúde mental $(16,4 \%)^{4}$

A adolescência apresenta-se como um momento do ciclo da vida repleto de situações em que as vulnerabilidades podem aflorar devido às singularidades e enfrentamentos dos desafios psicobiológicos, socioculturais e socioeconômicos que podem surgir..$^{4-5}$ Nesse sentido, torna-se relevante que o espaço educacional, com sua complexidade e conjuntura, aborde o adolescente de forma integral. É nesse ambiente que os escolares passam parte do seu dia, adquirindo e trocando conhecimentos para compreenderem o mundo em que vivem. A realização de ações de saúde nesse ambiente pode facilitar o processo de sensibilização, melhorar a assimilação e a capacidade de tomar decisões e, consequentemente, amenizar as vulnerabilidades. ${ }^{5}$

Diante desse cenário, considera-se relevante que, na agenda das políticas públicas voltadas para os adolescentes, estejam incluídas ações efetivas que venham a reduzir as condições de vulnerabilidade nesse grupo, e que haja uma maior sensibilização dos gestores no sentido de conhecerem as demandas de saúde dessa população, tendo em vista a melhoria da atenção à saúde nessa fase do desenvolvimento humano.

Apesar de não constituir um tema novo nas discussões sobre a saúde do adolescente, ainda existe uma escassez de investigações que contemplem em um único estudo as demandas de saúde desse grupo por meio da investigação dos determinantes do processo saúde-doença no contexto escolar. Nesse sentido o presente trabalho busca responder à pergunta: Há demandas de saúde entre adolescentes no município de Divinópolis, Minas Gerais? Desse modo, buscando contribuir para o aumento da produção científica a respeito do perfil e das demandas de saúde dos adolescentes e, consequentemente, auxiliar na ampliação das ações realizadas pelo sistema de saúde, com a adoção de ações protetoras e ofertas de práticas de assistência à saúde 
oportunas, este estudo objetivou identificar o perfil e as demandas de saúde de adolescentes escolares no município de Divinópolis, Minas Gerais.

\section{Método}

Trata-se de um estudo transversal de abordagem quantitativa, exploratório-descritivo, realizado em 2018 com 389 adolescentes do município de Divinópolis, Minas Gerais, Brasil.

O município foi selecionado para este estudo por ser de grande porte e por constituir-se sede da Região Ampliada Oeste de Minas Gerais. Quanto aos estabelecimentos e serviços de saúde, o município contava com cobertura de Estratégia Saúde da Família (ESF) de 50,76\% e 43 equipes atuantes na Atenção Primária à Saúde, sendo 32 operadas no modelo da ESF e 11 em unidades de atenção básica tradicionais. ${ }^{6}$

Em relação à Educação, o município possuía em 2017, 53 escolas, sendo 27 Estaduais e 26 Municipais, nas quais estavam matriculados 8.057 alunos nos $8^{\circ}$ e $9^{\circ}$ anos do Ensino Fundamental. ${ }^{6}$ Para o cálculo do tamanho amostral, considerou-se uma proporção de 50\% para desfechos de prevalência desconhecida, valor este que fornece o maior tamanho amostral para população finita, erro de 5\% e IC de 95\%, obtendo uma amostra representativa de 389 adolescentes que participaram do estudo e preencheram o questionário.

Os critérios de inclusão estabelecidos para a participação no estudo foram: estar regularmente matriculado no $8^{\circ}$ ou $9^{\circ}$ ano do Ensino Fundamental e não estar afastado das atividades escolares. Os critérios de exclusão compreenderam estar ausente no dia da coleta de dados e apresentar alguma condição que o impedia cognitivamente de responder às perguntas do questionário.

O critério de escolha de trabalhar com alunos do $8^{\circ}$ e $9^{\circ}$ ano se deu devido ao fato de que os alunos matriculados nessas séries se concentram, em sua maioria, na faixa etária de 12 a 15 anos, período em que é descrito na literatura como a fase de maior vulnerabilidade na 
5 | Silva GA, Ribeiro IKS, Silva HRM, Rezende TMRL, Belo VS, Romano MCC

adolescência. ${ }^{5}$ Os dados foram coletados por meio de um questionário estruturado e de autopreenchimento utilizado previamente em outro estudo, ${ }^{3}$ apresentando os seguintes módulos: caracterização socioeconômica (recebimento de Bolsa Família, trabalho na adolescência), saúde mental, uso de tabaco e álcool, uso de drogas, atividade física, violência, sexualidade e conhecimentos sobre Infecções Sexualmente Transmissíveis (ISTs), higiene, alimentação, relação com os pais, uso de tecnologias.

A análise estatística foi realizada com auxílio do Statistical Package of Social Sciences (SPSS) versão 21.0. Para comparações foram utilizados os testes Qui-quadrado de Pearson e Exato de Fisher na análise de variáveis categóricas. Os testes de Mann-Whitney e KruskallWallis foram empregados para a análise das variáveis numéricas que não apresentaram distribuição normal.

Foram atendidas todas as recomendações éticas sobre pesquisa com seres humanos contidas na Resolução № 466/2012 do Conselho Nacional de Saúde e o projeto de pesquisa foi submetido à análise e aprovado pelo Comitê de Ética em Pesquisa com Seres Humanos da Universidade Federal de São João Del-Rei, sob Parecer no 1.947 .282 , em 3 de março de 2017.

\section{Resultados}

Os participantes do estudo constituíram-se, em sua maioria, pelo sexo feminino, sendo 60,9\%. A média da idade foi de 13,8 anos, mediana de 14 anos, mínima de 12 anos e máxima de 17 anos. Sobre o ano escolar, predominaram os alunos do 9 $^{\circ}$ ano do Ensino Fundamental, sendo 67,5\%. A maior parte relatou não trabalhar $(86,4 \%)$ e 13,5\% informaram receber Bolsa Família.

Em relação ao consumo alimentar referente aos 30 dias anteriores a aplicação da pesquisa, 10,5\% dos alunos responderam que não comeram frutas, 10,3\% não ingeriram vegetais e 17,7\% informaram ter bebido refrigerante todos os dias. Quanto ao hábito de higiene corporal referente aos 30 dias anteriores à realização da pesquisa, 61,5\% dos adolescentes informaram 
escovar os dentes três ou mais vezes ao dia, 30\% afirmaram que na maioria das oportunidades lavavam as mãos antes das refeições, sendo que 62,8\% o faziam após a utilização do banheiro.

No que se refere à prática de atividade física nos últimos sete dias anteriores a investigação, 23,1\% dos participantes não realizaram exercícios físicos. Sobre o tempo de tela, ou seja, duração diária da utilização de aparelhos eletrônicos, como celular, computador, videogames, $20,8 \%$ dos adolescentes o fizeram durante três a quatro horas por dia, $11 \%$ cinco a seis horas por dia, $10,8 \%$ sete a oito horas e $14,1 \%$ utilizaram por mais de oito horas ao dia.

Acerca da experimentação, exposição e conhecimento dos adolescentes sobre álcool, 61,9\% dos meninos e 37,7\% das meninas relataram já ter experimentado bebida alcoólica, sendo que $35,2 \%$ declararam uso de bebida destilada e não destilada e 33,1\% consumiram álcool ao menos uma vez nos últimos 30 dias anteriores a investigação. A idade média de início de consumo de álcool foi 13,7 anos. A forma mais relatada de obtenção de bebidas foi por meio de amigos $(46,2 \%)$.

Sobre o uso de drogas ilícitas, identificou-se que 7,3\% dos adolescentes já haviam experimentado alguma dessas substâncias, sendo a maconha a mais popularizada (73,3\%). Ao serem questionados se seus pais fumam, todos os participantes do estudo disseram que seus pais não possuíam esse hábito. No entanto, grande parte dos adolescentes $(68,8 \%)$ mencionaram ter contato com pessoas fumantes.

Entre os participantes do estudo, no que se refere às relações interpessoais vivenciadas nos últimos 12 meses, as meninas foram as que se sentiram sempre sozinhas $(17,8 \%, \mathrm{p}=0,000)$ e que sempre estiveram preocupadas e não conseguiam dormir $(9,3 \%, p=0,000)$. Além disso, os meninos foram os que mais se envolveram em brigas $(5,3 \%, \mathrm{p}=0,021)$ (Tabela 1$)$.

No que tange à relação com os pais referente aos 30 dias anteriores a aplicação da pesquisa, 20,8\% dos adolescentes responderam que nunca se sentiram compreendidos e $10,5 \%$ 
destacaram que, durante o mesmo período mencionado anteriormente, os pais nunca sabiam onde os filhos se encontravam nos momentos vagos.

Tabela 1: Descrição dos adolescentes segundo relacionamentos interpessoais. Divinópolis, Minas Gerais. 2018, n= 389.

\begin{tabular}{|c|c|c|c|c|}
\hline \multirow{2}{*}{ Categoria } & \multicolumn{2}{|c|}{ Sexo } & \multirow[b]{2}{*}{ Total } & \multirow[b]{2}{*}{ Valor $\mathbf{p}$} \\
\hline & \multirow[t]{2}{*}{ Masculino } & \multirow[t]{2}{*}{ Feminino } & & \\
\hline Quantas vezes se sentiu & & & & $0,000^{\star}$ \\
\hline sozinho nos últimos 12 & & & & \\
\hline meses? & & & & \\
\hline Nunca & $53(34,9 \%)$ & $33(14 \%)$ & $86(22,2 \%)$ & \\
\hline Raramente & $53(34,9 \%)$ & $71(30,1 \%)$ & $124(32 \%)$ & \\
\hline Algumas vezes & $30(19,7 \%)$ & $61(25,8 \%)$ & $91(23,5 \%)$ & \\
\hline A maioria das vezes & $4(2,6 \%)$ & $29(12,3 \%)$ & $33(8,5 \%)$ & \\
\hline Sempre & $12(7,9 \%)$ & $42(17,8 \%)$ & $54(13,9 \%)$ & \\
\hline Quantas vezes se sentiu & & & & $0,000^{*}$ \\
\hline preocupado e não conseguiu & & & & \\
\hline dormir nos últimos 12 & & & & \\
\hline meses? & & & & \\
\hline Nunca & $60(39,7 \%)$ & $42(17,8 \%)$ & $102(26,4 \%)$ & \\
\hline Raramente & $41(27,2 \%)$ & $55(23,3 \%)$ & $96(24,8 \%)$ & \\
\hline Algumas vezes & $41(27,2 \%)$ & $84(35,6 \%)$ & $125(32,3 \%)$ & \\
\hline A maioria das vezes & $5(3,3 \%)$ & $33(14 \%)$ & $38(9,8 \%)$ & \\
\hline Sempre & $4(2,6 \%)$ & $22(9,3 \%)$ & $26(6,7 \%)$ & \\
\hline Possui quantos amigos & & & & $0,817^{*}$ \\
\hline íntimos? & & & & \\
\hline $\mathrm{Um}$ & $24(16,8 \%)$ & $48(20,8 \%)$ & $72(19,3 \%)$ & \\
\hline Dois & $37(25,9 \%)$ & $58(25,1 \%)$ & $95(25,4 \%)$ & \\
\hline Três & $77(53,8 \%)$ & $117(50,6 \%)$ & $194(51,9 \%)$ & \\
\hline Não possui amigo íntimo & $3(2,1 \%)$ & $3(1,3 \%)$ & $6(1,6 \%)$ & \\
\hline Frequência de agressões & & & & $0,381^{*}$ \\
\hline físicas no último ano. & & & & \\
\hline Nenhuma vez & $111(75,0 \%)$ & $190(82,3 \%)$ & $301(79,4 \%)$ & \\
\hline Uma ou Duas vezes & $23(15,5 \%)$ & $22(9,5 \%)$ & $45(11,9 \%)$ & \\
\hline Três ou Quatro vezes & $5(3,4 \%)$ & $6(2,6 \%)$ & $11(2,9 \%)$ & \\
\hline Cinco ou Seis vezes & $1(0,7 \%)$ & $3(1,3 \%)$ & $4(1,1 \%)$ & \\
\hline Sete ou Oito vezes & $2(1,4 \%)$ & $1(0,4 \%)$ & $3(0,8 \%)$ & \\
\hline Nove ou Dez vezes & $0(0 \%)$ & $2(0,9 \%)$ & $2(0,5 \%)$ & \\
\hline Onze ou mais vezes & $6(4,1 \%)$ & $7(3,0 \%)$ & $13(3,4 \%)$ & \\
\hline $\begin{array}{l}\text { Esteve envolvido em brigas } \\
\text { no último ano? }\end{array}$ & & & & $0,685^{*}$ \\
\hline
\end{tabular}




\begin{tabular}{lccc} 
Nenhuma vez & $92(61,7 \%)$ & $155(65,7 \%)$ & $247(64,2 \%)$ \\
Uma ou Duas vezes & $28(18,8 \%)$ & $41(17,4 \%)$ & $69(17,9 \%)$ \\
Três ou Quatro vezes & $12(8,1 \%)$ & $15(6,4 \%)$ & $27(7,0 \%)$ \\
Cinco ou Seis vezes & $6(4 \%, 0)$ & $6(2,5 \%)$ & $12(3,1 \%)$ \\
Sete ou Oito vezes & $4(2,7 \%)$ & $5(2,1 \%)$ & $9(2,3 \%)$ \\
Nove ou Dez vezes & $0(0 \%)$ & $4(1,7 \%)$ & $4(1,0 \%)$ \\
Onze ou mais vezes & $7(4,7 \%)$ & $9(3,8 \%)$ & $16(4,2)$ \\
Principal causa da lesão mais & & & \\
grave no último ano. & & & $0,021^{*}$ \\
Não teve lesões graves & $98(64,5 \%)$ & $189(81,1 \%)$ & $287(74,5 \%)$ \\
Acidente de automóvel & $7(4,6 \%)$ & $6(2,6 \%)$ & $13(3,4 \%)$ \\
Queda & $25(16,4 \%)$ & $20(8,6 \%)$ & $45(11,7 \%)$ \\
Algo caiu ou bateu em mim & $8(5,3 \%)$ & $5(2,1 \%)$ & $13(3,4 \%)$ \\
Brigas & $8(5,3 \%)$ & $7(3,0 \%)$ & $15(3,9 \%)$ \\
Outros motivos & $5(3,3 \%)$ & $6(2,6 \%)$ & $11(2,9 \%)$ \\
Quantas vezes sofreu assédio & & & \\
moral nos últimos 30 dias? & & & \\
Nenhuma vez & $120(78,9 \%)$ & $166(70,9 \%)$ & $286(74,1 \%)$ \\
Uma ou Duas vezes & $19(12,5 \%)$ & $41(17,5 \%)$ & $60(15,5 \%)$ \\
Três ou Quatro vezes & $3(2,0 \%)$ & $11(4,7 \%)$ & $14(3,6 \%)$ \\
Cinco ou Seis vezes & $3(2,0 \%)$ & $6(2,6 \%)$ & $9(2,3 \%)$ \\
Sete ou Oito vezes & $4(2,6 \%)$ & $3(1,3 \%)$ & $7(1,8 \%)$ \\
Nove ou Dez vezes & $0(0 \%)$ & $1(0,4 \%)$ & $1(0,3 \%)$ \\
Onze ou mais vezes & $3(2,0 \%)$ & $5(2,1 \%)$ & $8(2,1 \%)$ \\
\hline
\end{tabular}

${ }^{\star}$ Teste qui-quadrado

Quando indagados sobre a sexualidade, $20,2 \%$ dos adolescentes referiram já ter tido relação sexual, sendo maior o percentual entre os adolescentes do sexo masculino $(24,8 \%)$. Foi observado ainda que $28,9 \%$ dos que iniciaram atividade sexual relataram não utilizar nenhum tipo de método contraceptivo. A maioria dos adolescentes $(87,5 \% ; p=0,017)$ informaram que já ouviram falar sobre a infecção pelo HIV. Na auto avaliação sobre saúde, a maioria dos participantes considerou seu estado de saúde como ótimo ou bom $(84,4 \% ; \mathrm{p}=0,000)$ (Tabela 2).

Tabela 2: Descrição dos adolescentes pelos dados relacionados a vida sexual, conhecimento sobre HIV/Aids, situação vacinal e auto percepção sobre a saúde. Divinópolis, Minas Gerais, 2018, (n=389).

\begin{tabular}{|c|c|c|c|c|}
\hline \multirow[b]{2}{*}{ Categoria } & \multicolumn{2}{|c|}{ Gênero } & \multirow[b]{2}{*}{ Total } & \multirow{2}{*}{$\begin{array}{c}\text { Valor } \\
\mathbf{p}\end{array}$} \\
\hline & Masculino & Feminino & & \\
\hline
\end{tabular}


9 | Silva GA, Ribeiro IKS, Silva HRM, Rezende TMRL, Belo VS, Romano MCC

\begin{tabular}{|c|c|c|c|c|}
\hline Não & $112(75,2 \%)$ & $196(82,7 \%)$ & $308(79,8 \%)$ & \\
\hline $\operatorname{Sim}$ & $37(24,8 \%)$ & $41(17,3 \%)$ & $78(20,2 \%)$ & \\
\hline \multicolumn{5}{|l|}{$\begin{array}{l}\text { Idade que iniciou a vida } \\
\text { sexual }\end{array}$} \\
\hline$\leq 12$ anos & $13(34,2 \%)$ & $7(17,6 \%)$ & $20(100 \%)$ & \\
\hline$\geq 13$ anos & $25(65,7 \%)$ & $32(82,1 \%)$ & $57(100 \%)$ & \\
\hline Utiliza qual(is) método(s) & & & & $0,760^{*}$ \\
\hline \multicolumn{5}{|l|}{ contraceptivo atualmente? } \\
\hline Preservativo & $23(60,5 \%)$ & $20(52,6 \%)$ & $43(56,6 \%)$ & \\
\hline Hormônio Oral & $3(7,9 \%)$ & $4(10,5 \%)$ & $7(9,2 \%)$ & \\
\hline Preservativo + Hormônio & $2(5,3 \%)$ & $1(2,6)$ & $3(3,9 \%)$ & \\
\hline Não Utiliza & $10(26,3 \%)$ & $12(31,6 \%)$ & $23(30,2 \%)$ & \\
\hline Já ouviu falar sobre HIV/Aids & & & & $0,017^{*}$ \\
\hline Não & $26(17,65 \%)$ & $22(9,3 \%)$ & $48(12,5 \%)$ & \\
\hline $\operatorname{Sim}$ & $122(82,4 \%)$ & $215(90,7 \%)$ & $337(87,5 \%)$ & \\
\hline $\begin{array}{l}\text { A sua escola já abordou o } \\
\text { tema HIV/Aids }\end{array}$ & & & & $0,151^{*}$ \\
\hline Não & $25(16,9 \%)$ & $24(10,3 \%)$ & $49(12,8 \%)$ & \\
\hline Sim & $105(70,95 \%)$ & $183(78,2 \%)$ & $288(75,4 \%)$ & \\
\hline Não lembro & $18(12,2 \%)$ & $27(11,5 \%)$ & $45(11,8 \%)$ & \\
\hline \multicolumn{5}{|l|}{ HIV/Aids } \\
\hline Não & $105(71,4 \%)$ & $160(68,1 \%)$ & $265(69,4 \%)$ & \\
\hline $\operatorname{Sim}$ & $42(28,6 \%)$ & $75(31,9 \%)$ & $117(30,6 \%)$ & \\
\hline Situação Vacinal & & & & $0,578^{*}$ \\
\hline Em dia & $122(80,8 \%)$ & $188(79,7 \%)$ & $310(80,1 \%)$ & \\
\hline Em atraso & $2(1,3 \%)$ & $7(3,0 \%)$ & $68(17,6 \%)$ & \\
\hline Não sabe & $27(17,9 \%)$ & $41(17,4 \%)$ & $9(2,3 \%)$ & \\
\hline Vacina em atraso & & & & $0,549^{*}$ \\
\hline HPV & $2(22,2 \%)$ & $7(77,8 \%)$ & $9(100 \%)$ & \\
\hline Como você avalia sua saúde & & & & $0,000^{*}$ \\
\hline Ótima & $74(49,3 \%)$ & $67(28,5 \%)$ & $141(36,6 \%)$ & \\
\hline Boa & $64(42,7 \%)$ & $120(51,1 \%)$ & $184(47,8 \%)$ & \\
\hline Regular & $12(8,0 \%)$ & $46(19,6 \%)$ & $58(15,1 \%)$ & \\
\hline
\end{tabular}

${ }^{*}$ Teste qui-quadrado

\section{Discussão}

A presente investigação evidenciou que os adolescentes participantes do estudo apresentam demandas de saúde, especialmente no que se refere ao consumo alimentar inadequado, sedentarismo, utilização excessiva de tecnologias, uso de bebida alcoólica e drogas, 
saúde mental fragilizada, envolvimento em situações de violência e sexo inseguro. A complexidade das mudanças biopsicossociais que ocorrem na adolescência, além de características peculiares a essa fase do ciclo de vida, como a necessidade de ser aceito no grupo e a busca de identidade, promovem maior risco do adolescente assumir estilos de vida inadequados, como consumir drogas e se envolver em situações de violência. ${ }^{7-11}$

O elevado consumo de álcool entre os participantes do presente estudo se aproxima com a realidade do país. A Pesquisa Nacional de Saúde do Escolar - PeNSE ${ }^{4}$ revelou que 71,4\% dos escolares brasileiros do $9^{\circ}$ ano do Ensino Fundamental já experimentaram bebida alcoólica alguma vez na vida. Há uma popularidade do consumo de álcool entre adolescentes, iniciandose cada vez mais cedo. Nos Estados Unidos, a pesquisa de abrangência nacional Youth Risk Behavior Surveillance (YRBS) ${ }^{12}$ revelou que 70,8\% dos adolescentes consomem álcool.

Socialmente, o uso do álcool se configura como uma prática de inserção do indivíduo na comunidade. Cria-se, dessa forma, uma concepção sobre drogas, em que o adolescente passa a praticar o consumo para estabelecer laços sociais, estando mais susceptível aos amigos, à avaliação e aprovação, o que influencia sobremaneira o modo de como o adolescente se comporta. $^{12}$

Em 2012, investigação nacional que entrevistou cerca de 1.154 estudantes identificou a prevalência do uso de drogas ilícitas (maconha, inalantes ou cocaína) de 15,8\%, sendo maior a proporção de consumo de drogas entre adolescentes do sexo masculino (33\%), achado similar ao do presente estudo. ${ }^{12}$

Outro resultado relevante observado neste estudo refere-se aos hábitos alimentares dos adolescentes que ainda são inadequados, como, por exemplo, o consumo diário de refrigerante pelos alunos. Sabe-se que esta bebida é a que possui maior associação com o aumento do Índice de Massa Corporal (IMC), contribuindo com a atual epidemia da obesidade no Brasil e no mundo. $^{13}$ 
O ato de higienizar as mãos antes de comer e depois de ir ao banheiro e o hábito de preservar a saúde bucal, mostraram-se consolidados entre os escolares participantes deste estudo, corroborando os resultados encontrados pela PeNSE, ${ }^{4}$ que mostrou que essas práticas contribuem com a redução de casos de diarreia, transmissão de infecções de vias aéreas e presença de cárie entre os adolescentes.

No tocante as informações sobre o perfil da saúde mental, os achados revelaram que os adolescentes sentem-se solitários e preocupados. Esses dados são semelhantes aos encontrados na PeNSE ${ }^{4}$ e configura-se como uma importante adversidade a ser superada pela saúde pública, uma vez que o fator de solidão e preocupação com a privação do sono pode levar o adolescente a ideação suicida e ao suicídio consumado. ${ }^{14}$

Importante destacar que, na presente investigação, as meninas foram as que mais relataram sentimento de solidão e de preocupação, além da alteração do padrão do sono. Esses dados são semelhantes ao encontrado pelo Estudo de Riscos Cardiovasculares em Adolescentes (ERICA) que em 2016 mostrou que 30\% dos adolescentes brasileiros apresentam sintomas de ansiedade e depressão, especialmente entre as meninas $(38,4 \%) .{ }^{15}$

O presente estudo também analisou o tempo de tela, sendo constatado que grande parte dos adolescentes dedicou duas horas ou mais na realização de atividades como assistir televisão, jogar vídeo games e jogos de computador. A Pesquisa Health Behaviour in School-aged Children (HBSC), ${ }^{16}$ realizada com adolescente europeus e da América do Norte, constatou que $61 \%$ a $68 \%$ dos jovens entre 11 e 15 anos assistiam duas ou mais horas de televisão por dia.

Resultados similares foram encontrados na $\mathrm{PeNSE}^{4}$ que evidenciou que aproximadamente $60 \%$ dos escolares do $9^{\circ}$ ano do ensino fundamental assistiam televisão por duas ou mais horas diárias. O sedentarismo vem aumentando entre adolescentes nos últimos anos. De fato, o estudo ERICA $^{17}$ obteve resultados similares à presente pesquisa, na qual mais da metade dos participantes $(54,3 \%)$ são inativos em relação a atividade física. 
Corroborando os resultados relacionados à sexualidade nessa pesquisa, um estudo realizado em uma escola pública de um município de Rio Grande do Norte, identificou que $77,9 \%$ dos adolescentes relataram participar de atividades educativas com o tema ISTs e que em $31,4 \%$ dos casos quem conduziu essas atividades foram os professores da escola. Apesar do percentual relevante de adolescentes que já participaram dessas atividades, 68,6\% possuía conhecimento inadequado a respeito das formas de proteção e prevenção das ISTs. Esses dados evidenciam que mais importante do que propiciar acesso à temática é investir em estratégias de educação em saúde de qualidade, que possibilitam a contribuição de diferentes agentes e difusores de conhecimentos, sejam eles da educação ou da saúde. ${ }^{18}$

Chama atenção o percentual importante de adolescentes que relataram sexarca, o mesmo observado na pesquisa de Conhecimentos, Atitudes e Práticas na População Brasileira (PCAP) que identificou que a sexarca foi declarada por $77,6 \%$ dos jovens, e deste percentual, $30,9 \%$ dos estudantes da rede pública informaram início da atividade sexual antes dos 15 anos de idade. Além disso, a faixa etária dos 20 aos 39 anos é a mais atingida pela Aids no Brasil, e ao observar que a manifestação da doença pode ocorrer entre os 5 aos 15 anos após a contaminação, inferese que parte da população tenha adquirido a doença na adolescência, o que intensifica a importância da abordagem do tema entre a população jovem. ${ }^{18}$

Finalmente, se tratando dos dados apresentados sobre a vivência com situações de violência, de acordo com o Mapa de Violência (2016) do Brasil, há uma incidência de morte de 21,2/100 mil habitantes entre os adolescentes com 15 anos sendo mais prevalente entre os homens. Nessa investigação, a violência e assédio moral reforçam o achado da pesquisa de nível nacional, já que 15,5\% dos estudantes estiveram envolvidos em situações de agressão física uma ou mais vezes em um ano. ${ }^{19}$ 


\section{Conclusão}

Os resultados identificados confirmaram a hipótese deste estudo de que os adolescentes possuem demandas de saúde que não estão sendo devidamente assistidas pelos serviços de saúde. Aspectos relacionados à saúde mental fragilizada, uso de álcool e outras drogas, bem como consumo alimentar não saudável, sexo inseguro, sedentarismo, uso por tempo prolongado de eletrônicos e envolvimento com situações de violência foram identificadas como as demandas de saúde que necessitam de intervenções mais urgentes pelos profissionais de saúde e da educação.

A enfermagem possui papel importante na formulação de estratégias para assistir as demandas de saúde dos adolescentes pelo seu olhar holístico, assistência de forma humanizada, maior contato com os usuários da área de abrangência, responsabilidade pelas ações educativas e facilidade para construção do vínculo junto aos indivíduos assistidos. Essas características colocam o enfermeiro como agente essencial no processo de atenção as demandas de saúde dos adolescentes, que ainda são tidos como usuários “invisíveis”, devido ao fato de que, embora haja políticas públicas, inexistem, na práxis, ações assistenciais destinadas unicamente a esse grupo, no âmbito da atenção primária à saúde.

Importante destacar a necessidade da interprofissionalidade e intersetorialidade na Atenção Primária à Saúde no que se refere à assistência ao adolescente. Torna-se imperativa uma ação conjunta entre os serviços de saúde e as escolas. Os profissionais devem reconhecer o ambiente escolar como local mais propício para o acesso aos adolescentes e ter os profissionais da educação como aliados para as intervenções em saúde e identificação de adolescentes mais vulneráveis.

Assim sendo, a presente investigação não pretende generalizar os achados, uma vez que fazem parte de uma amostra de adolescentes de um município, mas cabe salientar que os resultados obtidos corroboraram com investigações nacionais e internacionais. Além disso, 
sugere-se a realização de outras pesquisas com delineamento metodológico de acompanhamento, como do tipo longitudinal, para identificação de fatores de risco relacionados à saúde dos adolescentes, visando romper a limitação de temporalidade de estudos seccionais.

Destaca-se que se torna importante o aprimoramento de políticas públicas especialmente no que tange à abordagem intersetorial das vulnerabilidades e das demandas apresentadas por esse grupo populacional. Essa perspectiva de integração é um pressuposto indispensável na abordagem e na construção de estratégias comuns para os atendimentos das demandas de saúde inerente ao adolescente.

\section{Referências}

1. Petersen CB, Lima RAG, Boemer MR, Rocha SMM. Necessidades de saúde e o cuidado de enfermagem. Rev Bras Enferm. 2016 [acesso em 2018 maio 18];69(6):1168-71. Disponível em: http://dx.doi.org/10.1590/0034-7167-2016-0128

2. Carvalho LPE, Lopes PN. O adolescente em vulnerabilidade social e a escola: uma análise sob a perspectiva histórico-cultural. Rev Juvent Políticas Públicas. 2018 [acesso em 2018 maio 18];2:1-13. Disponível em: https://revistasnj.mdh.gov.br/index.php/snj/article/view/93

3. Reis DC, Melo CPS, Soares TBC, Flisch TMP, Rezende TMRL. Vulnerabilidades e acesso à atenção primária à saúde na adolescência. Ciênc Cuid Saúde [Internet]. 2013 jan-mar [acesso em 2018 maio 18]; 12(1):063-071.

Disponível

em:

http://www.periodicos.uem.br/ojs/index.php/CiencCuidSaude/article/view/18141

4. Instituto Brasileiro de Geografia e Estatística (IBGE). Pesquisa nacional de saúde do escolar: 2015. Rio de Janeiro: IBGE; 2016. Disponível em: https://biblioteca.ibge.gov.br/visualizacao/livros/liv97870.pdf

5. Gomes AM, Santos MS, Finger DZA, Zanittini A, Franceschi V, Souza JB, et al. Refletindo sobre as práticas de educação em saúde com crianças e adolescentes no espaço escolar: um relato de extensão. Rev Conexão UEPG [Internet]. 2015 [acesso em 2018 maio 18];11(3):332-41. Disponível em: https://www.revistas2.uepg.br/index.php/conexao/article/view/7592

6. Ministério da Saúde (BR). CNES - Cadastro Nacional de Estabelecimentos de Saúde [Internet]; 2018 [acesso em 28 maio de 2018]. Disponível em: http://datasus.saude.gov.br/sistemas-e-aplicativos/cadastrosnacionais/cnes 
7. Brasil. Lei n. 9.069, de 13 de Julho de 1990. Dispõe sobre o Estatuto da Criança e do Adolescente e dá outras providências [Internet]. Diário Oficial da União, Brasília (DF); 1990 jul 16 [acesso em 2018 maio 18]. Seção 1, p. 1. Disponível em: http://www.planalto.gov.br/ccivil_03/leis/18069.htm\#art266

8. Ministério da Saúde (BR), Secretaria de Atenção em Saúde. Diretrizes nacionais para a atenção à saúde de adolescentes e jovens na promoção, proteção e recuperação da saúde. Brasília (DF): Ministério da Saúde; 2010.

9. Dallo LR, Martins RA. Associação entre as condutas de risco do uso de álcool e sexo desprotegido em adolescentes numa cidade do Sul do Brasil. Ciênc Saúde Colet [Internet]. 2018 jan [acesso em 2019 ago 23];23(1):303-14. Disponível em: http://www.scielo.br/scielo.php?script=sci_arttext\&pid=S141381232018000100303\&lng=en doi: http://dx.doi.org/10.1590/1413-81232018231.14282015

10. Andrade ME, Santos IHF, Souza AAM, Silva ACS, Leite TS, Oliveira CCC, et al. Experimentation with psychoactive substances by public school students. Rev Saúde Pública [Internet]. 2017 set [acesso em 2019 ago 23];51:82. Disponível em: http://www.scielo.br/scielo.php?script=sci_arttext\&pid=S003489102017000100273 doi: http://dx.doi.org/10.11606/s1518-8787.2017051006929

11. Centers for Disease Control and Prevention (CDC). Youth Risk Behavior Surveillance. Morb Mort Week Rep. 2012;61(4):168.

12. Cardoso LRD, Malbergier A. A influência dos amigos no consumo de drogas entre adolescente. Estud Psicol. 2014 mar [acesso em 2018 maio 18];31(1):65-74. Disponível em: http://www.scielo.br/scielo.php?script=sci_arttext\&pid=S0103-166X2014000100007 doi: http://dx.doi.org/10.1590/0103-166X2014000100007

13. Café ACC, Lopes CAOl, Novais RLR, Bila WC, Silva DK, Romano MCC, et al. Consumo de bebidas açucaradas, leite e sua associação com o índice de massa corporal na adolescência: uma revisão sistemática. Rev Paul Pediatr [Internet]. 2018 mar [acesso em 2019 aug 23];36(1):91-9. Disponível em: http://www.scielo.br/scielo.php?script=sci_abstract\&pid=S0103-

05822018000100091\&lng=en\&nrm=iso\&tlng=pt

14. Moreira LCO, Bastos PRHO. Prevalência e fatores associados à ideação suicida na adolescência: revisão de literatura. Psicol Esc Educ [Internet]. 2015 set-dez [acesso em 2018 ago 23];19(3):445-53. Disponível em: http://www.scielo.br/pdf/pee/v19n3/2175-3539-pee-19-03-00445.pdf

15. Lopes CS, Abreu GA, Santos DF, Menezes PR, Carvalho KMB, Cunha CF, et al. ERICA: prevalência de transtornos mentais comuns em adolescentes brasileiros. Rev Saúde Pública [Internet]. $2016 \mathrm{fev}$ [acesso em 2019 ago 23];50(Supl 1):14s. Disponível em: http://www.scielo.br/scielo.php?script=sci_arttext\&pid=S0034-89102016000200308\&lng=en doi: http://dx.doi.org/10.1590/s01518-8787.2016050006690 
16. World Health Organization (WHO), Regional Office for Europe. Inequalities in young people's health: HBSC international report from the 2005/2006 survey. Copenhagen: World Health Organization; 2008.

17. Cureau FV, Silva TLN, Bloch KV, Fujimori E, Belfort DR, Carvalho KMB, et al. ERICA: leisure-time physical inactivity in Brazilian adolescents. Rev Saúde Pública [Internet]. 2016 fev [acesso em 2019 ago 23];50(Supl 1):4s. Disponível em: http://www.scielo.br/scielo.php?script=sci_arttext\&pid=S003489102016000200304\&lng=en doi: http://dx.doi.org/10.1590/s01518-8787.2016050006683

18. Cordeiro JKR, Santos MM dos, Sales LKO et al. Adolescentes escolares acerca das dst/aids: quando o conhecimento não acompanha as práticas seguras. Rev Enferm UFPE On Line. 2017 jul [acesso em 2018 mai 18];11(Supl 7):2888-96. Disponível em: https://periodicos.ufpe.br/revistas/revistaenfermagem/article/view/9014/0

19. Ministério do Planejamento, Orçcamento e Gestão (BR). Mapa da Violência 2016: homicídios por arma de fogo no Brasil [Internet]. Brasília (DF): IPEA; 2016 [acesso em 2018 jul 04]. Disponível em: https://www12.senado.leg.br/institucional/omv/entenda-a-violencia/pdfs/atlas-da-violencia-2016

\section{Autor correspondente}

Nome: Márcia Christina Caetano Romano

E-mail: marciachristinacs@gmail.com

Endereço: Rua Sebastião Gonçalves Coelho, 400. Bairro: Chanadour. Divinópolis - MG. Brasil

CEP: $35.501-296$

\section{Contribuições de Autoria}

1 - Gabriela Aparecida da Silva

Participou da concepção e planejamento do projeto de pesquisa, obtenção e interpretação dos dados, redação e revisão crítica.

2 - Isabely Karoline da Silva Ribeiro

Participou da concepção e planejamento do projeto de pesquisa, obtenção e interpretação dos dados, redação e revisão crítica.

3 - Hugo Ricardo Moreira da Silva

Participou da concepção e planejamento do projeto de pesquisa, obtenção e interpretação dos dados, redação e revisão crítica.

4 - Tércia Maria Ribeiro Lima Rezende

Participou da redação e revisão crítica. 
17 | Silva GA, Ribeiro IKS, Silva HRM, Rezende TMRL, Belo VS, Romano MCC

5 - Vinicius Silva Belo

Participou do planejamento do projeto de pesquisa, análise e interpretação dos dados.

6 - Márcia Christina Caetano Romano (Orientadora)

Participou da concepção e planejamento do projeto de pesquisa, obtenção ou análise e interpretação dos dados, redação e revisão crítica.

\section{Como citar este artigo}

Silva GA, Ribeiro IKS, Silva HRM, Rezende TMRL, Belo VS, Romano MCC. Perfil e demandas de saúde de adolescentes escolares. Rev. Enferm. UFSM. 2019 [Acesso em: Anos Mês Dia];vol.9, e57: 1-17. DOI:https://doi.org/10.5902/2179769233510 